\title{
Clima de aprendizaje en el Residentado Médico
}

\author{
Educational climate in medical residency \\ Silvana Sarabia-Arce
}

\section{Resumen}

El clima de aprendizaje es un concepto que debe de ser relevante para todos aquellos involucrados en el proceso enseñanza-aprendizaje, es importante tanto para los alumnos como para los docentes de medicina, de pregrado y posgrado. Este artículo revisará las áreas involucradas en el clima de aprendizaje, los instrumentos empleados para evaluarlo y resaltará el impacto potencial del clima de aprendizaje para promover, estimular y optimizar el aprendizaje de los estudiantes y así mejorar la calidad de la experiencia educativa de los alumnos.

Palabras clave: Clima, aprendizaje, estudiantes, residencia médica.

\section{Abstract}

The educational climate is a concept that must be relevant to all of those involved in the teaching-learning process, it is important for both students and medical teachers. This article will review the areas involved in the educational climate, the tools used to assess it and will highlight the potential impact of the educational climate to promote, stimulate, optimize and improve the quality of the educational experience of the students.

Keywords: Climate, learning, students, medical residency.

\section{Introducción}

El clima organizacional es considerado una extensión lógica del clima psicológico. Específicamente, el clima organizacional se deriva de la agregación de las percepciones individuales del ambiente laboral, esto es, la forma típica que una persona en la organización le da significado a esa organización. Por lo tanto, el clima organizacional es el resultado de la suma de los climas psicológicos individuales ${ }^{(1)}$. Estos conceptos empleados por empresas y organizaciones desde hace muchos años, han sido más recientemente definidos como clima y ambiente de aprendizaje en las instituciones educativas.

El clima dentro del ambiente de aprendizaje (AA) es un concepto que debe de ser relevante para todos aquellos involucrados en el proceso enseñanza-aprendizaje, tanto para los alumnos como para los docentes de medicina, de pregrado y posgrado. El clima incluye, por ejemplo, cómo el ambiente puede estimular las actividades académicas e intelectuales y la medida en que puede fomentar la empatía, la cooperación y el apoyo. Este artículo revisará las áreas involucradas en el clima del AA, los instrumentos para evaluarlo y resaltará el impacto potencial del clima del AA para promover, estimular y optimizar el aprendizaje de los estudiantes y así mejorar la calidad de la experiencia educativa de los alumnos.
¿Cuál es la diferencia entre clima

y ambiente de aprendizaje?

La Real Academia Española (RAE) define ambiente como que rodea algo o a alguien como elemento de su entorno; conjunto de condiciones o circunstancias físicas, sociales, económicas, etc., de un lugar, una colectividad o una época; grupo o círculo social en que alguien se desarrolla o vive; disposición favorable o desfavorable de un conjunto de personas hacia alguien o algo ${ }^{(2)}$. Y, define clima como conjunto de condiciones atmosféricas de una región; ambiente ${ }^{(3)}$. La RAE los define como sinónimos; sin embargo, la diferencia radica en que el clima de aprendizaje involucra un componente subjetivo de la persona (alumno) y la actitud del grupo (profesor, administrativo u otro profesional de la salud) que recibe a esa persona ${ }^{(4)}$. Esto quiere decir que el ambiente puede ser uno y el clima, percibido de ese único ambiente, ser diferente dependiendo de cada individuo. Por ejemplo, una sala de operación es un ambiente único para todos aquellos los que están involucrados en una cirugía; pero, el clima de ese ambiente puede ser percibido de manera diferente por el cirujano principal, el ayudante, el instrumentista, el residente, el alumno, etc. Inclusive pueden estar las mismas personas involucradas en un ambiente y dependiendo de las circunstancias, cirugía complicada versus no complicada, el clima podría variar. Para algunos el clima podría considerarse como vago y 
etéreo, sin embargo, el efecto del clima es sustancial, real y muy influyente ${ }^{(5)}$.

El establecer un clima que favorezca el aprendizaje, la empatía, el trabajo en equipo y el bienestar del estudiante es sin duda una de las tareas más importantes que tiene todo profesor de medicina. Cabe señalar que en los artículos publicados sobre este tema los términos clima y ambiente pueden ser empleados de manera intercambiable; algunos diferenciarán clima y ambiente educacional ${ }^{(6)}$ y otros los emplearán como sinónimos ${ }^{(7)}$.

\section{Enseñando en un ambiente clínico}

Aprender y enseñar en un ambiente clínico puede ser una tarea compleja, demandante y frustrante que muchas veces se asume sin la preparación adecuada. Harden describe que el docente de medicina tiene ocho roles: ${ }^{\left({ }^{()}\right.}$

1. Proveedor de información y coach

2. Facilitador y mentor

3. Desarrollador curricular e implementador

4. Asesor y diagnosticador

5. Modelo a seguir como docente y clínico

6. Gerente y líder

7. Erudito e investigadory

8. Profesional.

Es evidente que para cumplir con todos los roles se requiere algo más que tener destreza clínica.

Con frecuencia el profesional que ha sido capacitado para ser básicamente un experto clínico a menudo debe desempeñar muchos roles simultáneamente, inclusive cambiando de un rol a otro en un mismo AA. Otra de las dificultades con las que se enfrenta el docente es el tiempo que puede dedicarle a la enseñanza. Si la responsabilidad clínica de un profesor aumenta, se reducirá, casi automáticamente, el tiempo que le dedica a hacer docencia ${ }^{(9)}$. Todos estos factores influenciarán directamente el AA.

Asimismo, es importante conocer y aplicar los principios del aprendizaje del adulto descritos por Knowles que se resumen a continuación: ${ }^{(10)}$

1. Establecer un clima de aprendizaje efectivo, donde los alumnos se sientan seguros y cómodos expresándose;

2. Involucrar a los estudiantes en la planificación;

3. Facilitar que los alumnos definan sus propias necesidades, lo que generará una motivación interna;

4. Estimular a los alumnos a que formulen sus propios objetivos de aprendizaje;

5. Incentivar a los alumnos a que identifiquen recursos y diseñen estrategias para lograr sus objetivos;

6. Apoyar a los alumnos en la realización de sus planes de aprendizaje; $y$,
7. Involucrar a los alumnos en la evaluación de su propio aprendizaje lo que ayudará a que desarrollen sus habilidades de reflexión crítica.

Todos los principios de la andragogía (el arte y la ciencia de ayudar a los adultos a aprender) son importantes; sin embargo, solo si se consigue el primer principio, esto es, establecer un clima de aprendizaje efectivo, se podrán lograr con mayor facilidad los otros principios.

\section{Un clima de aprendizaje saludable favorece el aprendizaje}

El aprendizaje en medicina está basado en la interacción de estudiantes de medicina y residentes con tutores, médicos, otros profesionales de la salud, pacientes y sus familiares; así, ningún médico en ningún país puede haber llegado a ser médico sin esta interacción ${ }^{(11)}$. Muchas veces son estas interacciones interpersonales las que llevan a que un AA adquiera "rasgos de personalidad" y sea descrito por alumnos, docentes, pacientes, personal de salud y otros, como: amigable, cálido, competitivo, agresivo, rígido, controlador, estricto, poco profesional, etc. ${ }^{(5)}$.

Si el objetivo de los programas de posgrado de medicina es preparar a los residentes para brindar una atención de calidad a los pacientes durante su capacitación y en su práctica futura es importante conocer que un clima de aprendizaje saludable contribuye a lograr una educación médica de alta calidad $^{(12)}$

\section{¿Cómo se evalúa el clima de aprendizaje?}

Para medir el clima de aprendizaje en posgrado de medicina se han empleado diversas escalas, tales como, Veteran affairs (VA) learners' perceptions survey, Learning environment assessment (LEA), Questionnaire from Rotem, Godwin and Du), Dundee Ready Education Environment Measure (DREEM), Operating room educational environment measure (OREEM), Surgical theatre educational environment measure (STEEM), Anaesthetic theatre educational environment measure (ATEEM), Ambulatory Care Learning Education Environment Measure (ACLEEM) y Postgraduate Hospital Educational Environment Measure (PHEEM) ${ }^{(13-21)}$. En una revisión sistemática que incluyó 79 estudios que evaluaron el clima de aprendizaje reportaron que se emplearon un total de 31 instrumentos. Diecinueve evaluaron el clima de aprendizaje en ambientes de medicina, 8 en enfermería y 4 en estomatología. Este estudio concluyó que la Dundee Ready Education Environment Measure (DREEM), Postgraduate Hospital Educational Environment (PHEEM), Clinical Learning Environment and Supervision y Dental Student Learning Environment Survey fueron probablemente los instrumentos más adecuados para evaluar el clima de aprendizaje en los alumnos de pregrado y posgrado de 
medicina, enfermería y estomatología respectivamente. Esta revisión recomienda que parte de las buenas prácticas educativas de una institución debe de ser el medir el clima de aprendizaje $^{(22)}$.

A continuación de describen brevemente los instrumentos de evaluación, PHEEM y ACLEEM, del clima de aprendizaje en el posgrado de medicina hospitalario $\mathrm{y}$ ambulatorio respectivamente.

\section{Medición del ambiente educacional hospitalario del posgrado}

La Postgraduate Hospital Educational Environment Measure (PHEEM) fue desarrollada por Roff et al. para evaluar el ambiente educacional hospitalario en posgrado de medicina ${ }^{(20)}$. Este instrumento consta de 40 ítems, tiene una confiabilidad alpha $>0,91$ y emplea una escala de Likert de 5 puntos, 4=completamente de acuerdo (CD), 3=de acuerdo (A), 2=inseguro (I), 1=en desacuerdo (D), $0=$ muy en desacuerdo (MD). Cuatro de los ítems ${ }^{(7,8,11,13)}$ son declaraciones negativas y deben de ser puntuadas de manera inversa, esto es, $4=\mathrm{MD}, 3=\mathrm{D}, 2=\mathrm{I}, 1=\mathrm{A}$ y $0=\mathrm{CD}$. La PHEEM evalúa tres subescalas: percepción del rol de autonomía (14 ítems), percepción de la enseñanza (15 ítems) y percepción del soporte social (11 ítems). Los puntajes de la escala pueden variar de 0-160. La siguiente es una guía para interpretar los resultados generales del clima de aprendizaje: 0-40=muy pobre, $41-80=$ muchos problemas, $81-120=$ más positivo que negativo, pero hay espacio para mejorar y $121-160=$ excelente. Esta escala ha sido empleada internacionalmente para evaluar el clima educacional en médicos en entrenamiento con una buena confiabilidad ${ }^{(23)}$. Una revisión sistemática reportada por Chan et al. revisó 30 estudios realizados en 14 países, y encontró diferencias en los puntajes de PHEEM según los diferentes niveles de entrenamiento, disciplinas y sitios de entrenamiento clínico ${ }^{(24)}$. También se observaron fortalezas y debilidades comunes en los entornos de aprendizaje. El estudio concluye que PHEEM se adopta ampliamente en diferentes entornos de aprendizaje y es una herramienta útil para identificar las fortalezas y debilidades de un entorno educativo.

\section{Ambulatory Care Learning Education Environment Measure (ACLEEM)}

La ACLEEM fue desarrollada y validada por Riquelme et al. para evaluar el AA ambulatorio en posgrado de medicina ${ }^{(20)}$. La validación incluyó 16 programas de residentado en varios países de Latinoamérica. La publicación incluye la versión de la escala en inglés y en español. Este cuestionario consta de 50 ítems, emplea una escala de Likert de cinco puntos, 4=completamente de acuerdo (CD), $3=$ de acuerdo (A), $2=$ inseguro (I), $1=$ en desacuerdo (D), $0=$ muy en desacuerdo (MD). Dos de los ítems (17 y 38) son declaraciones negativas y deben de ser puntuadas de manera inversa, esto es, $4=\mathrm{MD}, 3=\mathrm{D}, 2=\mathrm{I}, 1=\mathrm{A}$ y $0=\mathrm{CD}$. Los ítems fueron designados a tres dominios de la manera siguiente: Enseñanza clínica (ítems 1-16); Capacitación clínica (ítems 17-38); y Apoyo (ítems 39-50).

\section{Estudios sobre AA evaluados con PHEEM a nivel mundial}

En la tabla 1 se presenta un resumen de estudios sobre AA evaluados con PHEEM a nivel mundial.

\section{Encuesta Nacional de Médicos Residentes (ENMERE 2016)}

La ENMERE 2016 fue realizada por el Consejo Nacional de Residentado Médico del Perú (CONAREME) ${ }^{(32)}$. El objetivo de la encuesta fue describir las características laborales, académicas y de investigación de los médicos residentes en el 2016. Participaron 7393 médicos residentes, la edad promedio fue de $32,7 \pm 5,6$ años y el $42,9 \%$ fue de sexo femenino. Se halló menos que la mitad $(46,1 \%)$ de los médicos residentes estaban satisfechos con la función de su tutor y la mayoría $(80,9 \%)$ reportó no haber tenido descanso posguardia nocturna en el último mes. Aproximadamente 4 de cada 10 residentes $(38,6 \%)$ indicó haber recibido material de bioseguridad y solo el $16,1 \%$ había realizado al menos un trabajo de investigación durante su residencia. En relación a haber sufrido violencia alguna vez durante su residencia, se reportó violencia física, violencia verbal o amenaza y acoso sexual en $16,2 \%, 68,5 \%$ y $8,3 \%$, respectivamente. Estos resultados demuestran que hay alto porcentaje de percepción de maltrato en los médicos residentes. Este es el primer estudio publicado que describe las características laborales, académicas y de investigación del residentado médico en Perú. Es importante seguir realizando investigaciones que evalúen las condiciones del residente en el país, incluido el AA.

\section{La percepción del maltrato en la formación médica}

El maltrato durante la formación médica es un problema que ha sido reportado a nivel mundial ${ }^{(33)}$. Un metaanálisis realizado por Fnais et al. demostró que el 59,4\% de estudiantes de medicina han experimentado, por lo menos un tipo de acoso o discriminación durante su entrenamiento, siendo la agresión verbal la reportada con mayor frecuencia $(63,0 \%)^{(34)}$. Este último hallazgo es similar al encontrado en la ENMERE 2016.

La percepción del maltrato puede diferir entre individuos (por motivos personales, sociales, culturales, de género, etc.) $\mathrm{y}$, debido a esto, los comportamientos inadecuados y sus consecuencias deben de estar claramente definidas por las instituciones. Solo si identificamos el 


\begin{tabular}{|c|c|c|c|c|}
\hline \multicolumn{5}{|c|}{ Estudios sobre AA evaluados con PHEEM a nivel mundial } \\
\hline Autor/Año & País & $\mathbf{N}$ & $\begin{array}{l}\text { Programas } \\
\text { evaluados }\end{array}$ & Resultados \\
\hline $\begin{array}{l}\text { Clapham M, et al./ } \\
2007(25)\end{array}$ & $\begin{array}{l}\text { Inglaterra, } \\
\text { Escocia }\end{array}$ & 134 & $\begin{array}{l}9 \text { unidades de } \\
\text { cuidados intensivos }\end{array}$ & $\begin{array}{l}\text { Buen clima educacional, sin } \\
\text { racismo, ni sexismo, los } \\
\text { alumnos estaban contentos } \\
\text { con su enseñanza, el apoyo y } \\
\text { el trabajo que realizan }\end{array}$ \\
\hline \multirow[t]{2}{*}{$\begin{array}{l}\text { Vieira JE/ } \\
2008^{(26)}\end{array}$} & Brasil & 306 & $\begin{array}{l}\text { Medicina } \\
\text { Interna } \\
\text { Anestesiología } \\
\text { Cirugía }\end{array}$ & $\begin{array}{l}\text { Puntaje mayor en la subescala } \\
\text { de autonomía en medicina } \\
\text { interna. Puntaje mayor en la } \\
\text { subescala enseñanza en } \\
\text { anestesiología. }\end{array}$ \\
\hline & & & & $\begin{array}{l}\text { Puntajes similares en las tres } \\
\text { especialidades en la subescala } \\
\text { de soporte social. }\end{array}$ \\
\hline $\begin{array}{l}\text { Pinnock R, et al./ } \\
2009^{(27)}\end{array}$ & $\begin{array}{l}\text { Nueva } \\
\text { Zelanda }\end{array}$ & $\begin{array}{l}74(\mathrm{~B}) \\
68(\mathrm{~A})\end{array}$ & Pediatría & $\begin{array}{l}\text { Percepción positiva deL AA en } \\
\text { los alumnos de nivel básico } \\
\text { (B) y avanzado (A). }\end{array}$ \\
\hline $\begin{array}{l}\text { Herrera CA, et al./ } \\
2012^{(28)}\end{array}$ & Chile & 318 & $\begin{array}{l}35 \\
\text { especialidades }\end{array}$ & $\begin{array}{l}\text { No se encontraron diferencias } \\
\text { significativas entre género y la } \\
\text { universidad de origen. Los } \\
\text { extranjeros evaluaron mejor el } \\
\text { ambiente educativo que los } \\
\text { chilenos, no se percibió } \\
\text { racismo. }\end{array}$ \\
\hline $\begin{array}{l}\text { Shimizu T, et al)./ } \\
2013^{(29)}\end{array}$ & Japón & 206 & Medicina & $\begin{array}{l}\text { Un mejor ambiente educativo } \\
\text { en los hospitales mejorara el } \\
\text { rendimiento de los médicos } \\
\text { residentes. }\end{array}$ \\
\hline $\begin{array}{l}\text { Binsaleh S, et al/. } \\
2015^{(30)}\end{array}$ & $\begin{array}{l}\text { Arabia } \\
\text { Saudita }\end{array}$ & 38 & Urología & $\begin{array}{l}\text { Ambiente educacional menos } \\
\text { que satisfactorio }\end{array}$ \\
\hline $\begin{array}{l}\text { Koutsogiannou P, et al./ } \\
2015^{(31)}\end{array}$ & Grecia & 731 & $\begin{array}{l}33 \\
\text { especialidades }\end{array}$ & $\begin{array}{l}\text { La autonomía y el apoyo } \\
\text { social se observaron } \\
\text { ligeramente menor a lo } \\
\text { esperado mientras que lo } \\
\text { observado en enseñanza fue } \\
\text { mayor de lo esperado. }\end{array}$ \\
\hline
\end{tabular}

problema, solo si reconocemos que todos debemos ser parte activa para solucionar este problema, solo así encontraremos todos juntos, alumnos, residentes, docentes y autoridades las acciones a seguir para finalmente erradicar el maltrato en las escuelas de medicina. Es responsabilidad de todos hacer que la cultura institucional apoye la exigencia académica pero que sea intolerante con el maltrato en cualquiera de sus formas ${ }^{(11)}$.

\section{Promoviendo el bienestar}

Los médicos en formación tienen un alto riesgo de depresión y suicidio, siendo este riesgo notablemente más elevado si se compara con la población general. Una revisión sistemática y meta-análisis encontró una mayor prevalencia de suicidio entre los profesionales médicos, siendo el mayor riesgo de médicos y médicas de $40 \%$ y de $130 \%$, respectivamente, al compararlos con individuos de la misma edad y género de la población general ${ }^{(35)}$. Es por esto que a nivel mundial se están realizando esfuerzos para mejorar el bienestar del residente ${ }^{(36)}$. Uno de los aspectos que señalan como muy importantes en el bienestar del residente es el AA. Cuando se les consultó a los residentes cuáles serían las características ideales del AA en momentos de estrés refirieron cinco puntos: 1 . Conocer y desestigmatizar los problemas de salud mental; 2. Camaradería entre los residentes; 3 . Tutorías 
por asistentes y residentes superiores (dar retroalimentación positiva); 4. Disponibilidad de servicios de salud mental (consejería confidencial); y 5. Una cultura de apoyo ${ }^{(37)}$.

Es importante generar, en todas las instituciones involucradas en la capacitación de residentes, una cultura institucional que promueva los cambios que favorezcan un AA saludable, que identifique y maneje los problemas de salud mental en los residentes, de manera confidencial, que apoye el bienestary que proteja a los residentes.

\section{Profesionalismo}

El profesionalismo debe de ser considerado dinámico y determinado en términos conductuales y no solo como un conjunto de ideales escritos en un documento. Debe ser un acercamiento a la práctica diaria de la medicina que se expresa en comportamientos observables ${ }^{(38)}$. El profesionalismo debe fundamentarse en el compromiso que tiene un médico hacia su paciente, la profesión y la sociedad, esto es, las conductas, los conocimientos, la obligación de seguir aprendiendo y la interacción con los pacientes, sus familiares, los alumnos y los colegas $^{(39)}$.

La palabra profesionalismo debe tener un significado particular para los médicos, debe implicar todo lo que se admira en nuestros colegas y lo que habría que esforzarse en lograr ${ }^{(40)}$. Un buen AA definitivamente favorecerá el profesionalismo que es una competencia requerida para todos los residentes de todas las especialidades y, asimismo, para todos los médicos en ejercicio.
Referencias bibliográficas

1. James LR, Choi CC, Ko CHE, McNeil PK, Minton MK,

2. Real Academia Española. Ambiente. Diccionario de la Lengua Española.

Real Academia Española. Clima. Diccionario de la Lengua Española.

3. Riquelme Pérez A, Fuentes G, Jeria A, Méndez I, Aranís C,

4. Larios G, et al. Ambiente educacional y calidad de la docencia en la escuela de medicina. Ars Medica. 2007;36(2). https://arsmedica.cl/index.php/MED/article/view/149

5. Genn JM, Harden RM. What is Medical Education Here Really Like? Suggestions for action research studies of climates of medical education environments. Med Teach. 1986;8(2):111-124.

6. Genn JM. AMEE Medical Education Guide No. 23 (Part 1): Curriculum, environment, climate, quality and change in medical education< a unifying perspective. Med Teach. 2001;23(4):337-344.

7. Roff S, McAleer S, Ifere OS, Bhattacharya S. A global diagnostic tool for measuring educational environment: Comparing Nigeria and Nepal. Med Teach. 2001;23(4):378382.

8. Harden RM, Lilley P. The eight roles of the medical teacher. The purpose and function of a teacher in the healthcare professions. 1a Edición. Elsevier; 2018.

9. Ramani S, Leinster S. AMEE Guide no. 34: Teaching in the clinical environment. Med Teach. 2008; 30: 347-364.

10. Kaufman DM. ABC of learning and teaching in medicine. Applying educational theory in practice. BMJ. 2003;326:213216.

11. Sarabia S. La percepción del maltrato en la formación médica. Rev Neuropsiquitr. 2018;81(3):135-140.

12. Silkens MEWM, Lombarts KMJMH, Scherpbier AJJA, Heineman MJ, Arah OA. Towrdas healthy learning climates in postgraduate medical education: exploring the role of hospital-wide education committees. BMC Medical Education. 2017;17:241-248.

13. Kaminetzky CP, Keitz SA, Kashner TM, Aron DC, Byrne JM, Chang BK, et al. Training satisfaction for subspecialty fellows in internal medicine: Findings from the Veterans Affairs (VA) Learners' Perceptions Survey. BMC Med Education. 2011;11:21-30.
14. Roth LM, Severson RK, Probst JC, Monsur JC, Markova, T, Kushner SA, et al. Exploring physician and staff perceptions of the learning environment in ambulatory residency clinics. Fam Med. 2006;38(3):177-184.

15. Rotem A, Godwin P, Du J. Learning in hospital settings. Teach Learn Med. 1995;7(4)211-217.

16. Roff S, McAleer S, Harden RM, Al-Qahtani M, Ahmed AU, Deza $\mathbf{H}$ et al. Development and validation of the Dundee Ready Education Environment Measure (DREEM). Med Teach. 1997;19(4):295-299.

17. Diwwadkar GB, Jelovsek JE. Measuring surgical trainee perceptions to assess the operating room educational environment. J Surg Educ. 2010;67(4):210-216.

18. Dimoliatis IDK, Jelastapulu E. Surgical Theatre (Operating Room) Measure STEEM (OREEM) Scoring overestimates educational environment: the 1-to-L Bias. Universal J Educ Res. 2013;1(3):247-254.

19. Holt MC, Roff S. Development and validation of the Anaesthetic Theatre Educational Environment Measure (ATEEM). Med Teach. 2004;26(6):553-558.

20. Riquelme A. Padilla O, Herrera C, Olivos T, Roma JA, Sarfatis A, et al. Development of ACLEEM questionnaire, an instrument measuring residents' educational environment in postgraduate ambulatory setting. Med Teach. 2013;35(1):e861-e866.

21. Roff S, McAleer S, Skinner A. Development and validation of an instrument to measure the postgraduate clinical learning and teaching educational environment forhospital-based junior doctors in the UK. Med Teach. 2005;27(4):326-331.

22.Soemantri D, Herrera C, Riquelme A. Measuring the educational environment in health professions studies: A systematic review. Med Teach. 2010;32(12):947-952.

23. Wall D, Clapham M, Riquelme A, Vieira J, Cartmill R, Aspegren K, Roff S. Is PHEEM a multi-dimensional instrument? An international perspective. Med Teach. 2009;31(11):e521-e527.

24. Chan CY W, Sum MY, Lim WS, Chew NWM, Samarasekera DD, Sim K. Adoption and correlates of Postgraduate Hospital Educational Environment Measure (PHEEM) in the evaluation of learning environments - A systematic review. Med Teach. 2016;38(12):1248-1255. 
25.Clapham M, Wall D, Batchelor A. Educational environment in intensive care medicine--use of Postgraduate Hospital Educational Environment Measure (PHEEM). Med Teach. 2007;29(6):e184-e191.

26. Vieira JE. The postgraduate hospital educational environment measure (PHEEM) questionnaire identifies quality of instruction as a key factor predicting academic achievement. Clinics (Sao Paulo). 2008;63(6):741-746.

27.Pinnock R, Reed P, Wright M. The learning environment of paediatric trainees in New Zealand. J Paedriatr Child Health. 2009;45(9):529-534.

28.Herrera CA, Olivos T, Román JA, Larraín A, Pizarro M, Solís $\mathbf{N}$, et al. Evaluación del ambiente educacional en programas de especialización médica. Rev Med Chile. 2012; 140: 1554-1561.

29.Shimizu T, Tsugawa Y, Tanoue Y, Konishi R, Nishizaki Y, Kishimoto M, et al. The hospital educational environment and performance of residents in the General Medicine In-Training Examination: a multicenter study in Japan. Int J Gen Med. 2013;6:637-640.

30.Binsaleh S, Babaeer A, Alkhayal A, Amadbouly K. Evaluation of the learning environment of urology residency training using the postgraduate hospital educational environment measure inventory. Adv Med Educ Pract. 2015; 6: 271-277.

31.Koutsogiannou P, Dimoliatis IDK, Mavridis D, Bellos S, KarathanosV, Jelastopulu E. Educational Environment Measure (PHEEM) in a sample of 731 Greek residents. BMC Res Notes. 2015;8:734-746.

32. Consejo Nacional de Residentado Médico, Escobedo- Palza S, Nieto-Gutierrez W, Taype-Rondan A, Timaná-Ruiz R,
Alva-Diaz C, et al. Características del residentado médico en el Perú: resultados de la primera Encuesta Nacional de Médicos Residentes (ENMERE-2016). Acta Med Peru. 2017;34(4):273-282

33. Chávez-Rivera A, Ramos-Lira L, Abreu-Hernández LF. A systematic review of mistreatment in medical students. Gac Med Mex. 2016;152:711-725.

34. Fnais NS, Soobiah C, Hong Chen M, Lillie E, Perrier L, Tashkhandi M, et al. Harassment and discrimination in medical training: A systematic review and meta-analysis. Acad Med. 2014;89:817-827.

35. Schernhammer ES, Colditz GA. Suicide rates among physicians: a quantitative and gender assessment (metaanalysis). Am J Psychiatry. 2004;161(12):2295-2302.

36. Silkens MEWM, Chahine S, Lombarts KMJMH, Arah OA. From good to excellent: Improving clinical departments learning climate in residency training. Med Teach. 2017;40(3);237-243.

37.Daskivich TJ, Jardine DA, Tseng J, Correa R, Stagg BC, Jacob KM, Harwood JL. Promotion of wellness and mental health awareness among physicians in training: Perspective of a national, multispecialty panel of residents and fellows. J Grad Med Educ. 2015;7(1):143-147.

38. Lesser CS, Lucey CR, Egener B, Braddock CH, Linas SL, Levinson W. A behavioral and systems View of proffessionalism. JAMA. 2010;304(24):2732-2737.

39. Harolds $\mathbf{J}$. What is professionalism and how can we promote it? Clin Nucl Med. 2011;36(6):457-459.

40.Sarabia S. Profesionalismo, medicina y psiquiatría. Rev Neuropsiquiatr. 2017;80(1):247-248.

Citar como: Sarabia-Arce S. Clima de aprendizaje en el Residentado Médico. Diagnóstico (Lima). 2019;58(2):132-137.

DOI: 10.33734/diagnostico.v58i3.59

Correspondencia: Silvana Sarabia-Arce Correo electrónico:silvana.sarabia@upch.pe

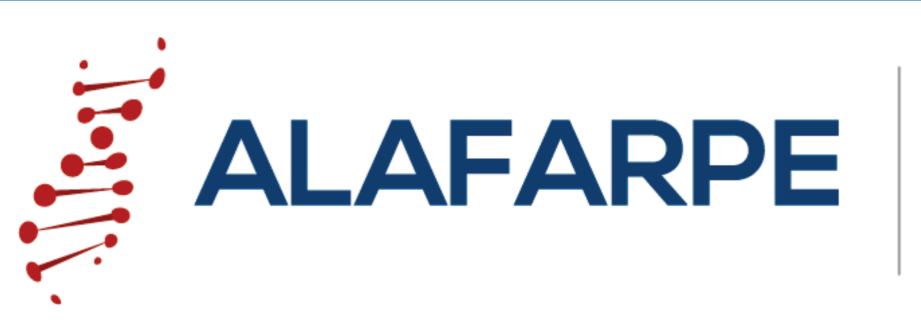

\section{ASOCIACIÓN NACIONAL DE LABORATORIOS FARMACÉUTICOS}

\section{CORREO ELECTRÓNICO: \\ alafarpe@alafarpe.org.pe}

WEB:

www.alafarpe.org.pe 